\title{
Effects of textural and surface characteristics of metal-organic frameworks on the methane adsorption for natural gas vehicular application
}

Jingmei Shen, ${ }^{\mathrm{a}^{*}}$ Jacqueline Sulkowski, ${ }^{\mathrm{a}}$ Matthew Beckner, ${ }^{\mathrm{a}}$ Anne Dailly ${ }^{\mathrm{b}}$

${ }^{a}$ Optimal CAE Inc., Plymouth, MI 48170, USA

${ }^{\mathrm{b}}$ General Motors Global Research and Development Center, Warren, MI 48090, USA

*Corresponding author. Email: Jingmei.shen@gm.com, phone: +1- 586-986-2189, fax: +1- 586-986-2244

\begin{abstract}
In this work, methane adsorption and textural and surface characteristics of selected 46 microporous metal-organic frameworks and 6 other adsorbents were measured experimentally. The objective of this work is to identify either the most relevant characteristics or a combination of multiple properties, which will qualify a given sample to be a good methane uptake material in a moderate pressure range (up to 70 bar) and at $298 \mathrm{~K}$. It is found that there is an overall linear tendency between maximum excess methane adsorption and BET specific surface area. The micropore volume correlates to the maximum excess methane adsorption as well, irrespective of the chemistry and functionalities of materials. In addition, micropore size distribution has an impact on methane uptake. When considering the total methane uptake, special attention should be paid to the effect from packing density. The
\end{abstract}


evaluation also focuses on the discussion of deliverable capacity and concludes that optimal adsorption enthalpy is desired to avoid large amount of methane retained at the minimum desorption pressure in practical vehicular applications.

\section{Keywords}

Natural gas/Methane adsorption, Metal-organic frameworks, Adsorption isotherms, Surface characteristics, Natural gas vehicles

\section{Introduction}

With rising oil prices and uncertainty about security of oil supplies, the diversification of energy resources for the automotive transportation sector has become of particular importance not only in North America, but in other countries around the world [1]. A promising alternative fuel is natural gas (NG). NG is a gaseous mixture of hydrocarbons, predominantly methane (about 70 to $90 \% \mathrm{CH}_{4}$ ). A typical NG composition from pipeline also contains carbon dioxide, nitrogen, oxygen and hydrogen sulphide $[2,3]$. Advantages of NG compared to gasoline are its relatively low price and wide availability. $80 \%$ to $90 \%$ of the natural gas used in US is domestically produced as it can be drawn from wells or extracted in conjunction with crude oil production. An extensive network of natural gas pipelines is already in place to deliver fuel directly to many sites including individual homes. In addition, NG is a clean-burning alternative fuel. However only $\sim 0.1 \%$ of NG in US is used for transportation fuel currently.

One of the challenges of using NG in automotive is the energy content of NG per unit volume of

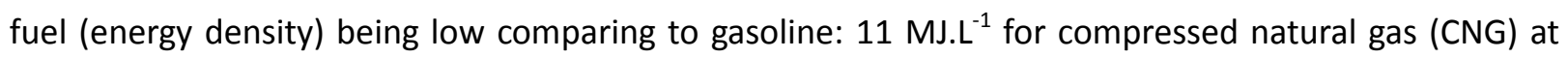


24.8 MPa $(3,600 \mathrm{psi})$ vs $32 \mathrm{MJ}^{-1}$ for gasoline [4,5]. A CNG fuel tank would need to be approximately three times larger than a gasoline tank to allow a vehicle the same driving range. Currently, CNG is mainly stored in onboard tanks under high pressure at 250 bar service pressure in the United States and 200 bar service pressure in the rest of the world $[6,7]$. The high pressure vessels for CNG storage are constrained in their geometry, typically cylindrical, and relatively heavy weight (about $1 \mathrm{~kg}$ per liter for steel tank). High pressure CNG also requires costly multi-stage compression [9].

These issues could be addressed by using the adsorbed natural gas (ANG) technology. With ANG technology, NG is reversibly adsorbed on the surface of sponge-like materials without any chemical bonding. Fuel can be stored at relatively low pressures, 35 bar. The lower pressure design will allow the use of conformable and less expensive storage options containing advanced new material alternatives $[2,7,9,10]$.

Considerable research and development are still required to move ANG to market. In-depth study of the adsorption fundamentals must be performed. The key component of the ANG technology is the adsorbent material. The adsorbents' evaluation and comparison are usually conducted by measuring the excess gravimetric gas adsorption at $(P, T)$, that is, the difference between the amount of adsorbate stored in the adsorbed region and the amount that would be present in an identical volume in the absence of solid-gas interactions. The total volumetric adsorption, including all the gas molecules in the pores and defect voids, is then determined in order to compare to the CNG benchmark technology $[4,10,11]$. In addition, for vehicular NG storage system applications, the parameter known as "deliverable capacity" or "usable capacity" is most relevant for comparing the performances of different adsorbents. This "deliverable capacity" is defined as the amount of gas that is released from 
the adsorbent when the pressure is reduced to a certain pressure from the service pressure [4]. 2 to 5 bar is a pressure range commonly cited as minimum desorption pressures, since the vehicle engine requires a minimum inlet NG pressure to operate $[3,12,13]$. Therefore, the deliverable capacity of an ANG system is always lower than the total storage capacity, usually by around $15 \%$ but sometimes by as much as $30 \%$ [4].

The porous adsorbent materials that have been reported in the literature for methane adsorption are essentially microporous: zeolites [14], activated carbons $[8,15,16]$, porous organic polymer networks $[3,11]$, and metal-organic frameworks (MOFs) $[3,11]$. Among these materials, MOFs are a relatively new family of microporous materials for gases sorption applications. For traditional carbons or zeolites materials, numerous works agree that methane adsorption uptake is favored by high surface areas, high micropore volumes, and average pore sizes within the range of $8-15 \AA[5,15,19]$. Considering MOF materials, more factors such as open metal sites, porosity, topologies and framework densities could be taken into account.

Even though there have been several studies reporting on how each of those factors affects the methane storage uptake using either an experimental [12,20-22] or a simulation method $[13,23-26]$, few reports have discussed the impact of the combination of the variable properties of MOFs on the material storage performances. A general comparison between MOF materials and other microporous adsorbents is yet to be established. Düren et al. performed a simulation study on the adsorption characteristics of methane in several isoreticular MOFs (IRMOF), molecular squares, zeolites, crystalline porous silica, and carbon nanotubes. They found a correlation between the methane adsorption at 35 bar and $298 \mathrm{~K}$ with the surface area and proposed new IRMOF structures as potential adsorbents [27]. 
Wang investigated the methane adsorption in nine MOF materials with different topologies using simulation methods as well. Similarly to Düren's work, the results showed that the specific surface area is more important than other properties of MOFs for methane adsorption at $298 \mathrm{~K}$ and moderate pressure [28]. Just recently, Long et. al. summarized total methane adsorption data reported in literature for MOFs and compared to experimental data obtained in their laboratory on the six most promising MOFs [3]. These reports and many other comparative studies rely on data from different sources and publications.

Herein, methane uptake and other physical properties, such as material porosity and density, of 52 microporous adsorbents including 4 carbons, 2 polymer materials, and 46 MOFs have been measured in our laboratory experimentally. The objective of this work is to identify either the most relevant characteristics or a combination of multiple properties, which will qualify a sample to deliver the required methane storage at $298 \mathrm{~K}$ and pressures up to 70 bar. All adsorption data were collected from two identical PCT instruments, which minimized the effects of instrument and data analysis methodology differences on the adsorption results. This is a major difference from most reported comparative studies of MOFs based on data from public sources. The selected materials in this work cover a large range of surface functionalities, pore structures and BET surface areas. Comparing with the previous investigations mentioned above, our study also encompasses more potentially important factors. We also include the issue of "deliverable capacity" or "usable capacity" in our discussion and its relationship with open metal sites and pore sizes.

\section{Experimental Methods}




\subsection{Materials}

Various adsorbents have been explored as potential methane storage materials, among them are activated carbons, porous polymer networks (PPN), and metal-organic frameworks (MOFs). The adsorbents investigated in this study were either synthesized in house, obtained from numerous collaborations with Universities (Texas A\&M University, University of Nottingham, University of California Berkeley, and University of Michigan), or bought from suppliers. All materials were provided along with their crystalline characterization.

\subsection{Material characterizations}

The air and moisture sensitive samples were handled, loaded, and weighed in an inert atmosphere (argon) on a precision balance $( \pm 1 \mathrm{mg})$. The remaining samples were loaded and weighed in a room temperature atmosphere on a precision balance $( \pm 0.1 \mathrm{mg})$. Additionally, all materials were outgassed in the sample holder at various temperatures and a $10^{-5}$ torr vacuum overnight prior to any sorption measurements. The analysis of the porous texture of the adsorbents was carried out using $\mathrm{N}_{2}$ and $\mathrm{Ar}$ adsorption measurements at $77 \mathrm{~K}$ and $87 \mathrm{~K}$, respectively, using a Quantachrome Autosorb-1 gas-sorption apparatus. The samples were degassed at $373 \mathrm{~K}$ under vacuum overnight. The specific surface area (SSA) was determined from $\mathrm{N}_{2}$ at $77 \mathrm{~K}$ using the Brunauer-Emmett-Teller (BET) equation. It should be noted that the SSA was calculated over a range of P.P. ${ }^{-1}$ of $5 \times 10^{-2}-1.0 \times 10^{-1}$, as these conditions can influence substantially the value of the resulting area. The pore size distribution was calculated from the Ar sorption data using a standard non-local density function theory (NLDFT) method for MOFs and PPNs. The total pore volume $V_{p}$ was obtained from the saturation value of $\mathrm{Ar}$ adsorption isotherm (0.995 P.P. $\left.{ }^{-1}\right)$ measured at $87 \mathrm{~K}$ using liquid $\mathrm{Ar}$ density. The micropore volume $\mathrm{V}_{\mathrm{m} . \mathrm{p}}$ was calculated from the Ar sorption data or $\mathrm{N}_{2}$ sorption data using Dubinin-Raduschkevich (DR) method 
[29].

Volumetric methane adsorption measurements at ambient temperature were performed using an automated volumetric Sieverts' apparatus (PCT-Pro 2000 from Hy-Energy LLC) over the range of 0 to 70 bar. The excess methane adsorption isotherms were calculated from successive gas expansions. The dead space volumes were determined at $298 \mathrm{~K}$ using helium gas as a negligibly adsorbing gas. Ultra-high purity methane and helium (99.999\% purity) obtained from Airgas Inc. were used for all measurements.

\subsection{Data analysis}

The excess adsorption is the only quantity associated to the adsorbed phase readily accessible to measurements $[4,30]$. Herein, the excess methane adsorption is measured on a gravimetric basis as the amount of excess methane per unit weight (gravimetric excess), namely $G_{e x}$. Using the volumetric method (i.e. Sieverts' method), $G_{e x}$ can be calculated from successive gas expansions by summing the differences between the amounts of gas depleted from the reference cell and that occupying the sample cell after each expansion such as:

$$
G_{e x}=\sum_{j}^{k} \Delta G_{j}-\rho_{f}^{k} V_{d}
$$

Where $\Delta G$ is the amount of gas sent to a cell containing the adsorbent after an expansion, $\rho=P / Z R T$ is the gas density and $V_{d}$ is the so-called dead-space volume of the sample cell. $V_{d}$ is, in fact, directly measured with helium as a non-adsorbing gas and includes all the free volume accessible to the methane in the sample cell.

In order to accurately measure the excess adsorption, the selected material is tested at least two times to ensure the isotherm is reproducible and consistent. 
The total gravimetric methane adsorption $\left(G_{t o t}\right)$ differs from $G_{e x}$ as it accounts for the total amount of adsorbate molecules present in the bulk volume of the material. $G_{\text {tot }}$ is the convolution of all the methane stored in the adsorbing space (i.e. absolute adsorption) and in the non-adsorbing space (i.e. gas compressed in interparticular voids and macropores). $G_{\text {tot }}$ can be calculated from $G_{e x}$ according to the following equation:

$$
G_{\text {tot }} \quad=\quad G_{\text {eX }}+\rho_{g} \quad \times \quad V_{V}
$$

Where $\rho_{g}$ is the density of the bulk gas and $v_{v}$ is the total void volume of the adsorbent. The void volume $V_{v}$ is obtained from the bulk density $\left(\rho_{b}\right)$ and the skeletal density $\left(\rho_{\text {ske }}\right)$ of the adsorbent using:

$$
V_{V}=\left(1 / \rho_{b}\right)-\left(1 / \rho_{s k e}\right)
$$

The total volumetric methane adsorption can then be expressed as follows:

$V_{\text {tot }} \quad G_{\text {tot }} \quad \times \quad \rho_{b}$

(Eq. 4)

Considering that the skeletal and bulk densities of an adsorbent can differ by up to an order of magnitude, it is clear that $G_{\text {tot }}$ can be much higher than $G_{e x}$. Although the adsorbed excess amount is the quantity directly measureable, $V_{\text {tot }}$ is the important quantity to take into account for engineering perspectives and technology comparison. The bulk density should be used for total adsorption calculations because it includes the contribution of the particle volume, the interparticle void volume, and internal pore volume. Contrary to the crystallographic density, the bulk density is not an intrinsic 
property of the material and it can change depending on how the material is handled. However when the bulk density is not available, the crystallographic density, which excludes all voids that are not a fundamental part of the molecular arrangement, can be used. MOFs, offering a clear structural platform, allow for use of the crystallographic density. This leads to an ideal maximum estimate of a material's total adsorption capacity. 


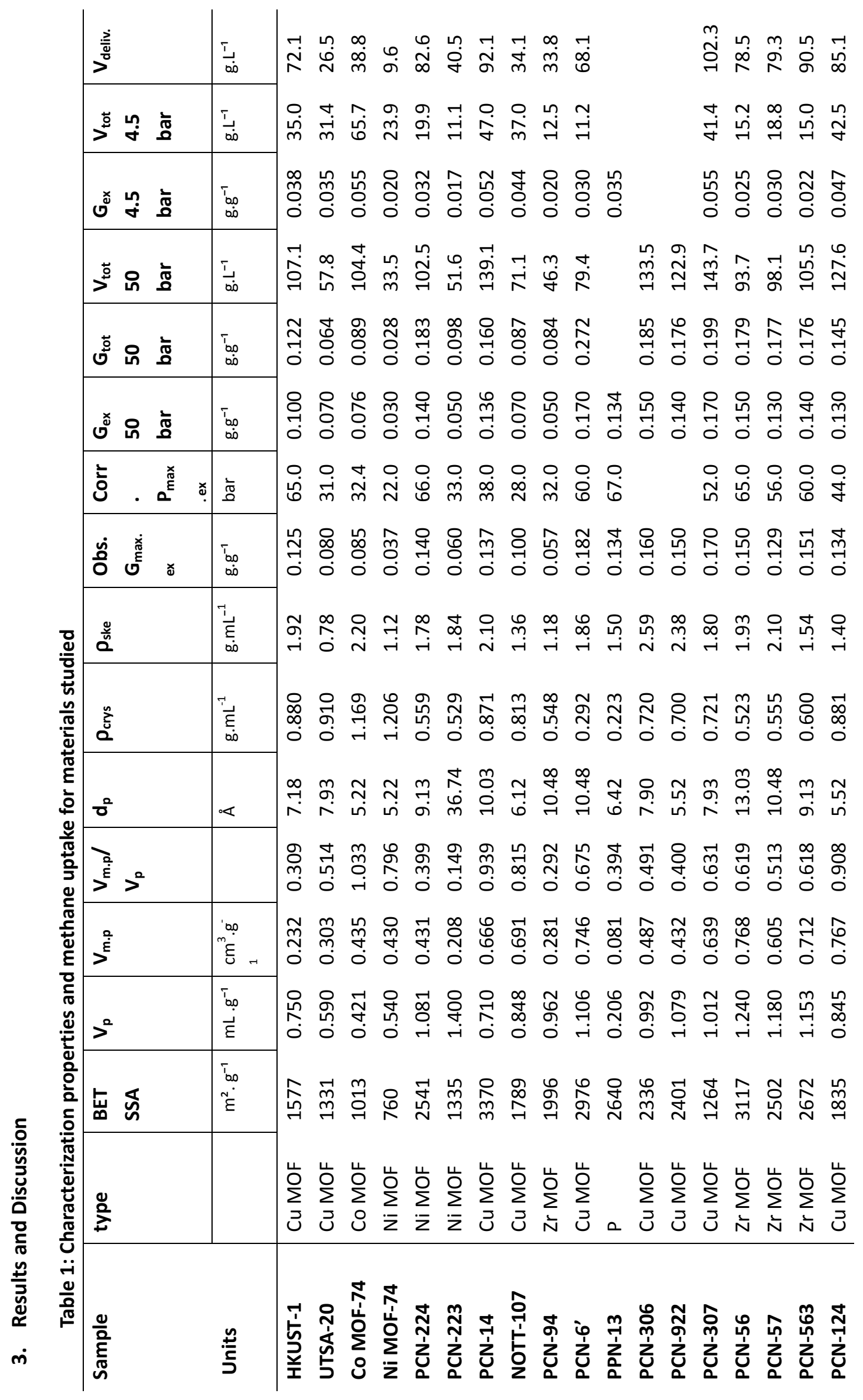




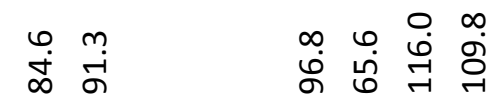

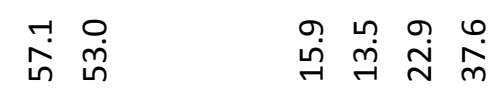

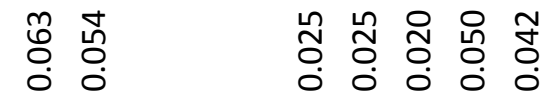

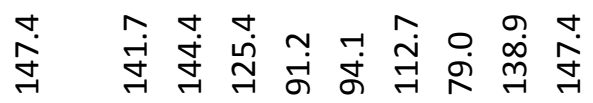

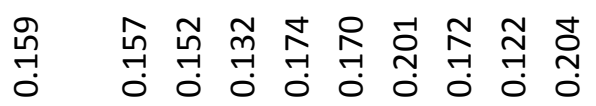

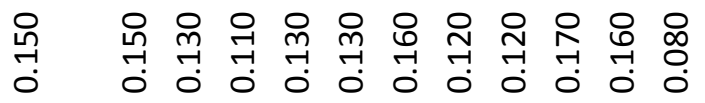

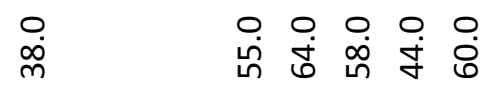

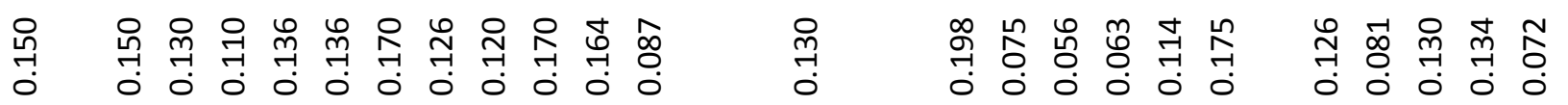

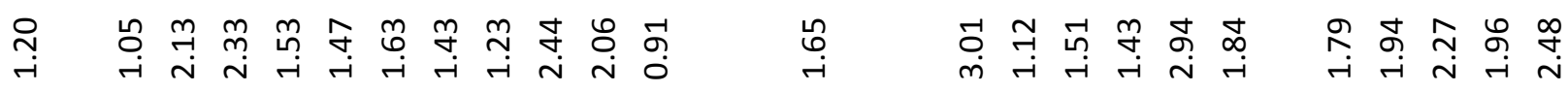

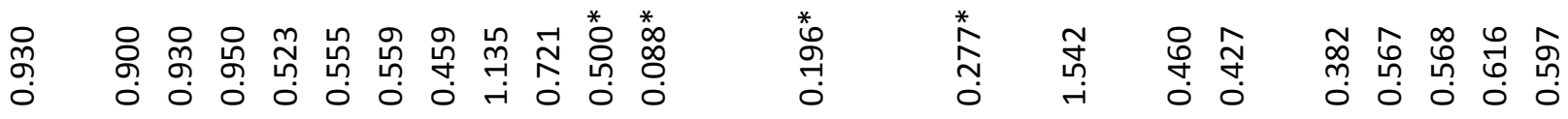

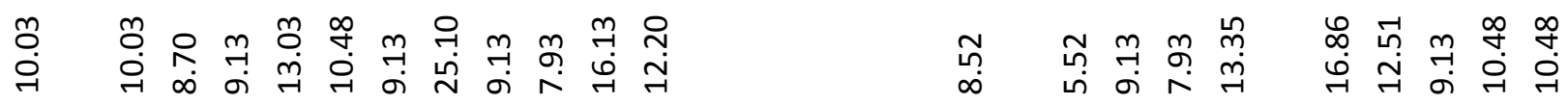

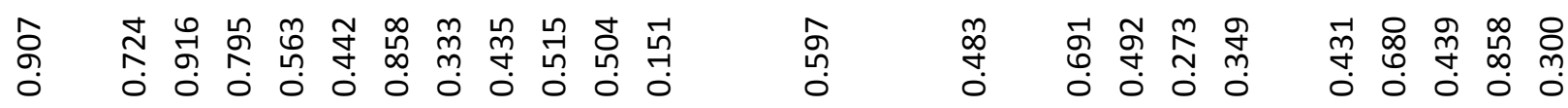

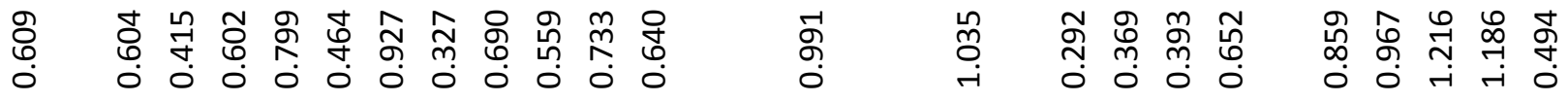

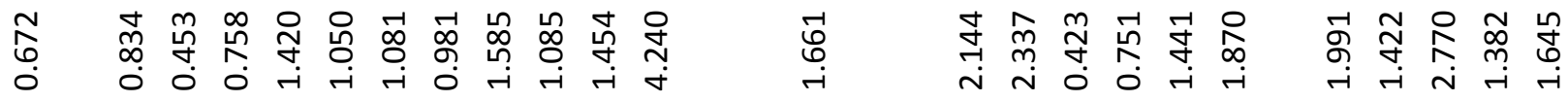

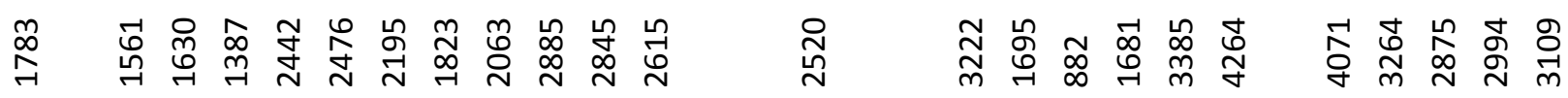

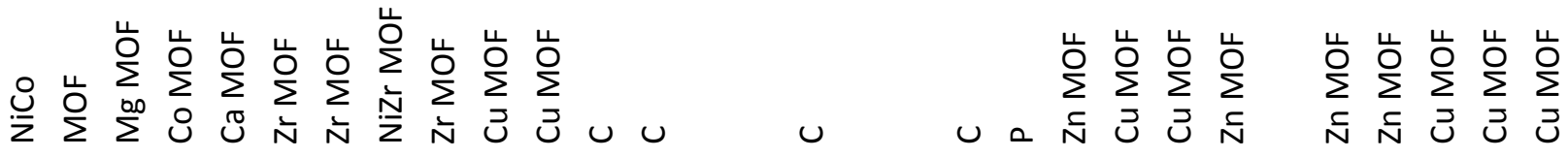

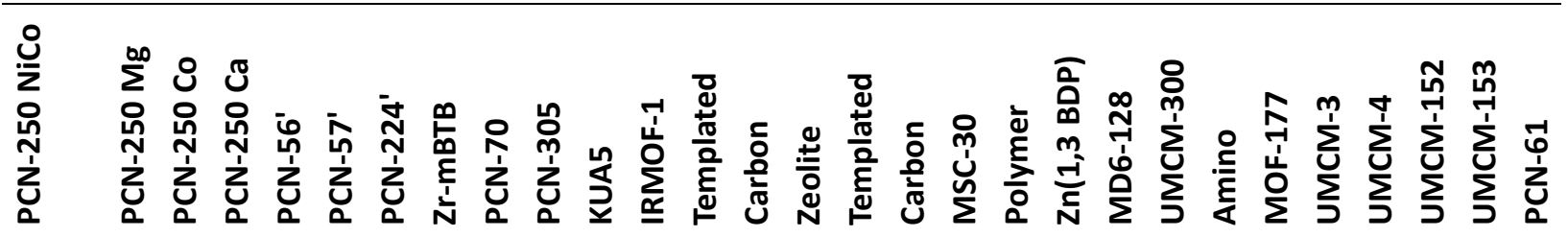




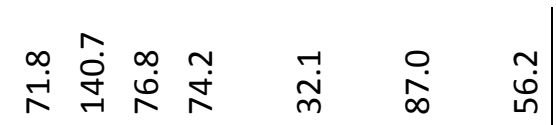

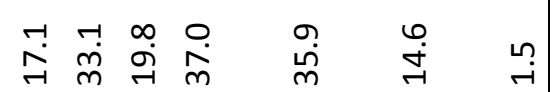

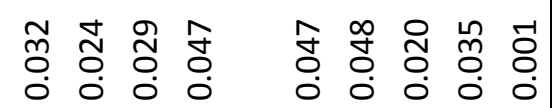

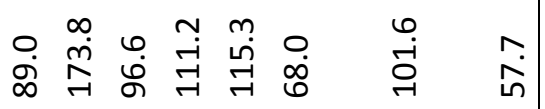

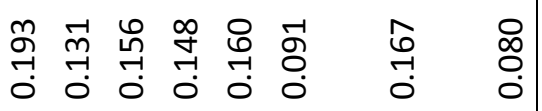

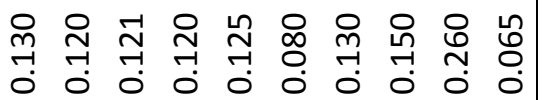

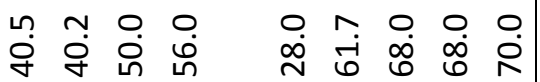

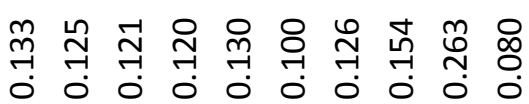

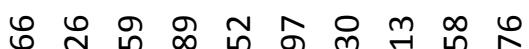

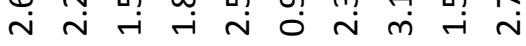

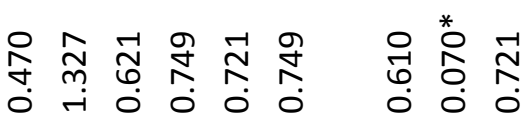

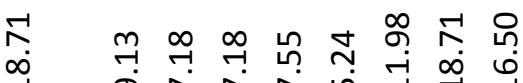

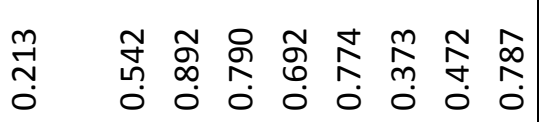

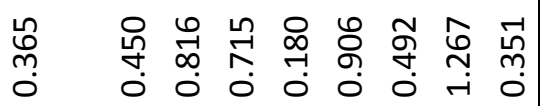

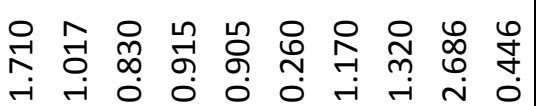

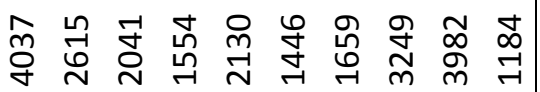

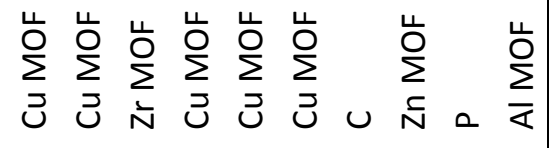

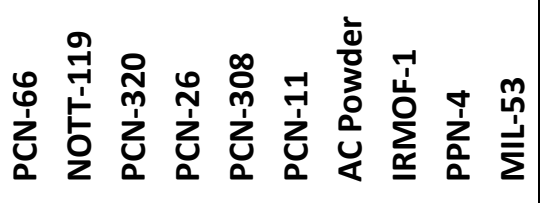




\subsection{Comparison of gravimetric maximum excess methane adsorptions at $298 \mathrm{~K}$ and pressure up to 70 bar}

Excess methane adsorption uptake $G_{e x}$ can be measured directly and is the most important characterization parameter for adsorbents. The presence of a maximum is a typical feature of excess adsorption isotherms. At higher pressures, the adsorbed phase density inside the material's pores starts to saturate while the bulk gas density keeps increasing. The maximum and following rollover in the isotherm occur when the density of the coexistent bulk phase increases faster than the adsorbed phase.

From the reported adsorption data in literatures the following properties influence the excess adsorption uptakes: accessible surface area, pore volume, pore size, and adsorbent/guest affinity (adsorption energy). In this study, almost all the materials have an average pore width in the range of 8-15 Å. Table 1 summarizes our experimental characterizations including the maximum excess $\mathrm{CH}_{4}$ uptakes, total $\mathrm{CH}_{4}$ uptakes calculated from crystallographic density at 4.5 and 50 bar, the resulting deliverable $\mathrm{CH}_{4}$ capacities, and textural characteristics (BET SSA, total pore volume $V_{p}$, micropore volume $V_{m . p}$, average pore width $d_{p}$, crystal density $\rho_{\text {crys, }}$ and skeletal density $\left.\rho_{\text {ske }}\right)$. The metal ions used in MOF structures are also listed here in order to evaluate their potential influence on the methane sorption performance of the material. Note also that for most of the studied MOFs materials the porosity activation was conducted in the laboratory and could lead to some discrepancy in final results compared to already published data. In addition, it comes to our attention that in a number of instances, there was no indication whether published methane adsorption data were given in terms of excess, total, or absolute adsorption. This could also lead to potential disagreement when comparing our results with 
published data $[3,14]$.

The relations between the physical properties of the adsorbents in Table 1 and the maximum excess methane adsorptions have been examined. Our methane sorption measurements are performed over the entire pressure range, from vacuum to saturation, to more fully characterize these promising high surface area materials. As shown in Fig. 1 (a) the maximum excess methane uptake, $G_{\text {max.ex }}$, and the BET specific surface area have a linear correlation (red dash line in Fig. 1(a)). We also set up the "tolerance band" as shown between two orange dash lines. Most of the plots fall in the band (blue diamonds). This occurs regardless of the adsorbents' chemistry or functionality. The maximum excess methane uptake correlates more strongly with the micropore volume $V_{m . p}$ than it does with the total pore volume $V_{p}$, as shown in Fig. 1 (b) and Fig. S1. This is consistent because any pores of a larger dimension will not contribute significantly to the overall methane adsorption performances of a predominantly microporous adsorbent. The greatest variation of $G_{\text {max.ex }}$ vs. $V_{p}$ is shown for materials which are most likely to have broader pore size distribution. This can explain the greater overall scatter presented in the pore volume data.

Taking a closer look at the textural characteristics of these potential natural gas adsorbents, we found that samples outside (red diamonds) or on the edge (green diamonds) of the tolerance band in Fig. 1(a), by having lower methane uptakes than that predicted from the linear correlation with BET SSA, display relatively low micropore volume $V_{m . p}$ (pore width $<20 \AA$ ), compared with other samples with similar surface areas. From Table 1 , it is also shown that samples with low ratios of $V_{m . p}$ to $V_{p}$ have relatively low methane uptakes. For instance, PCN-94, PCN-61, and PCN-66 whose maximum excess 
adsorption uptakes are lower than expected have ratios of $0.292,0.300$, and 0.213 , respectively. On the contrary, samples with higher methane uptakes than that predicted from the linear correlation with BET SSA usually display relatively high ratios of $V_{m . p}$ to $V_{p}(>0.5)$.

Besides of having the large proportion of micropores, samples showing higher uptakes than that predicted from the correlation also show a narrow micropore size distribution These samples contain a large proportion of micropores with pore sizes which are believed to be with the optimum pore size range for methane adsorption, around $8 \AA$ [1]. On contrast, those with a broad pore size distribution or relatively large average pore width have lower methane uptakes than those read from the line in Fig. 1(a). As shown in Fig. 1(a), PCN-307 (red diamond) has higher methane uptake than that predicted from the linear correlation between the maximum excess uptakes and BET SSA data. PCN-70 (blue diamond) falls right on the line. UMCM-3 (red diamond) and UMCM-300 (green diamond) are below the line, having lower methane uptake than that expected from the linear correlation.

Fig. 2 depicts the comparison of the cited materials' micropore size distributions. PCN-307 has mainly a narrow single peak with the average pore width of $7.93 \AA$. PCN-70 with the average pore width of $9.13 \AA$ h has one major peak centered at about $8 \AA$, but several small peaks with pore width between 15 and $20 \AA$, indicating the existence of some larger micropores. The pore size distribution plot of UMCM-3 present two large narrow peaks centered at around 16 and $27 \AA$, respectively, thus the average pore width is $16.86 \AA$, much larger than the theoretical optimum pore size of $8 \AA$ for methane adsorption. UMCM -300 has an average pore width of $7.9 \AA$, but also a broad distribution of pore sizes between 8 and $27 \AA$. The results of these four samples clearly indicate that although surface area and micropore 
volume are the two major factors to affect the $G_{\text {max.ex }}$, average pore widths and pore size distributions should also be considered, while the adsorbents chemistry and functionalities are not showing a great impact under these test conditions.

In fact, even for the samples in the tolerance band (blue diamonds), ones having similar values of BET SSA can differ by $50-100 \%$ in $G_{\text {max.ex }}$ in Fig. $1($ a). In most cases, that can be explained by comparing tothe data of micropore volume $V_{m . p}$ (Table 1 and Fig. $\left.1(b)\right)$. Samples with low $V_{m . p}$ have low $G_{\text {max.ex }}$ generally. For example, PCN-223 (Ni) with SSA of $1335 \mathrm{~m}^{2} . \mathrm{g}^{-1}$ has much lower $V_{m . p}$ of PCN-223 (Ni), 0.149 $\mathrm{mL} \cdot \mathrm{g}^{-1}$ comparing to PCN-250 Ca with SSA of $1387 \mathrm{~m}^{2} \cdot \mathrm{g}^{-1}\left(V_{m . p}=0.795 \mathrm{~mL} \cdot \mathrm{g}^{-1}\right)$. We can see that the $G_{\text {max.ex }}$ of PCN-223 (Ni), $0.050 \mathrm{~g} / \mathrm{g}$, is also much lower than that of PCN-250 Ca, $0.11 \mathrm{~g} / \mathrm{g}$, though the adsorption enthalpy of MOFs with Ni should be higher than that of MOFs with $\mathrm{Ca}$. The metal effect is not significant here. In some complicated cases, other factors like the portion of micropores, and pore size have to be considered as described above. MD6-128 $\left(1681 \mathrm{~m}^{2} \cdot \mathrm{g}^{-1}\right)$, AC powder $\left(1659 \mathrm{~m}^{2} \cdot \mathrm{g}^{-1}\right), \mathrm{PCN}-250$ Co (1630 $\left.\mathrm{m}^{2} \cdot \mathrm{g}^{-1}\right)$, and PCN-250 Mg $\left(1561 \mathrm{~m}^{2} \cdot \mathrm{g}^{-1}\right)$ with similar BET SSA have $G_{\text {max.ex }}$ of $0.063,0.126,0.13$, and 0.15 g/g, respectively. The $G_{\text {max.ex }}$ of AC powder and PCN-250 Co are twice of that of MD6-128. PCN-250 Mg has even better performance. As shown in Table 2, MD 6-128 with the lowest methane uptake has the lowest $V_{m . p}, 0.369 \mathrm{~mL} . \mathrm{g}^{-1}$, and the lowest $V_{m . p}$ to $V_{p}$ ratio, 0.492 . AC powder has the highest $V_{m . p}, 0.906$ $\mathrm{mL} \cdot \mathrm{g}^{-1}$, but its average pore size is only $5.24 \AA$, too small for methane uptake. PCN-250 Co and PCN-250 Mg are isostructrual with different metal centers. Herein, again metal effect is not obvious. Even though PCN-250 Co should have higher methane adsorption enthalpy than PCN-250 Mg, PCN-250 Mg with higher $V_{m . p}$ displays higher $G_{\text {max.ex. }}$ 
Table 2: Characterization properties and methane uptakes for MD6-128, AC Powder, PCN-250 Co, and PCN-250 Mg

\begin{tabular}{|l|l|l|l|l|l|l|}
\hline Sample & type & $\begin{array}{l}\text { BET } \\
\text { SSA }\end{array}$ & $\mathbf{V}_{\text {m.p }}$ & $\begin{array}{l}\mathbf{V}_{\text {m.p }} / \\
\mathbf{V}_{\mathbf{p}}\end{array}$ & $\mathbf{d}_{\mathbf{p}}$ & $\begin{array}{l}\text { Obs. } \\
\mathbf{G}_{\text {max. }} \\
\text { ex }\end{array}$ \\
\hline Units & & $\mathrm{m}^{2} \cdot \mathrm{g}^{-1}$ & $\mathrm{~cm}^{3} \cdot \mathrm{g}^{-1}$ & & $\AA$ & $\mathrm{g}^{\mathrm{g}} \mathrm{g}^{-1}$ \\
\hline MD6-128 & Cu MOF & 1681 & 0.369 & 0.492 & 9.13 & 0.063 \\
AC Powder & $\mathrm{C}$ & 1659 & 0.906 & 0.774 & 5.24 & 0.126 \\
PCN-250 Co & Co MOF & 1631 & 0.415 & 0.916 & 8.7 & 0.13 \\
PCN-250 Mg & Mg MOF & 1561 & 0.604 & 0.724 & 10.03 & 0.15 \\
\hline
\end{tabular}

Another way to ponder the excess methane uptakes vs. the textural properties of the materials, is to consider the pressure at which the material reaches its maximum adsorption value, $P_{\max . e x}$. Experimental excess adsorption data clearly exhibit maxima. As mentioned earlier this is a typical feature of excess adsorption isotherms. At low pressures, the adsorbed phase density is much higher than the bulk density of methane. Both densities increase with pressure but the bulk density increases more rapidly than the adsorbed phase density and eventually at the maximum, both densities are increasing at the same rate so that pressure has no effect on the excess amount adsorbed. At even higher pressure the bulk density continues to increase with pressure while the adsorbed phase density approaches a saturation condition where no more molecules can fit into the pores. The excess adsorption is zero at the pressure where the bulk and adsorbed phase densities are equal. Therefore, we can expect that the pressure at which the material reach its maximum excess adsorption directly relates to its pore volume. 
Additionally, $P_{\max . e x}$ also reflects the interaction potential between the adsorbate molecules and the walls of the pores, also described as the adsorption energy. A strong adsorption energy results in a steep initial slope of the isotherm curve at low pressure, in accordance with Henry's Law adsorption. If the adsorption energy is weaker the slope of the isotherm does not look as steep and the $P_{\max , e x}$ moves to higher pressures. The pore size and pore size distributions, surface functionalities, and open metal sites for MOFs, as the common factors affecting adsorption energy have been discussed extensively in the literature.

Fig. 3 shows that for most of our samples (blue diamonds), there is a linear trend between the total pore volume $V_{p}$ and the $P_{\text {max.ex }}$. This is consistent with the expectation described above, and suggest that those samples feature similar adsorption enthalpies. No obvious trends between either the BET surface area and $P_{\text {max.ex }}$ or $V_{\text {m.p }}$ and $P_{\text {max.ex }}$ are observed in Fig. S2 (a) and (b), respectively. Specifically for MOFs, since metal centers have been identified as the primary methane adsorption sites, the adsorption energy is expected to vary depending on the constitutive metal center ions. However, the fact that most MOFs with different metal ions fall on the line in Fig. 3 indicates the low impact from the metals on adsorption energy, under the experimental conditions, i.e. up to 70 bar. We conclude that metal centers have a strong influence on the adsorption energy at low coverage but do not strongly affect the adsorption energy at the maximum adsorbate uptake. That is consistent with the previous conclusion from the discussion of the $G_{\text {max.ex. }}$.

The few samples (red diamonds) not falling on the line in Fig. 3, such as PPN-4, MSC-30, and Polymer display lower maximum pressure than predicted from the linear correlation. They all feature 
broad pore size distributions with relatively small portion of micropores. The MOF samples displaying lower than expected maximum pressure, UMCM-3 $(\mathrm{Zn})$ and UMCM-152 (Cu), exhibit average pore widths of $16.86 \AA$ and $9.13 \AA$, respectively. UMCM-152 features a broad pore size distribution as well as a $V_{p}$ of $2.77 \mathrm{~mL} . g^{-1}$ and a $V_{m . p}$ of $1.216 \mathrm{~mL}^{-1}{ }^{-1}$ (ratio of $V_{m . p}$ to $V_{p}$ is 0.439 ). The influence from the adsorption energy to the $P_{\text {max.ex }}$ is observed here, since large pores cause weaker interactions between the adorbate molecules and the pore walls.

\subsection{Comparison of total methane adsorptions at $298 \mathrm{~K}$ and $50 \mathrm{bar}$}

35 bar is a typical benchmark for the maximum achievable pressure of common single-stage compressors and is often used in the evaluation of total methane adsorptions at room temperature in literature $[3,11,14]$. Nevertheless, herein most of the adsorption isotherms measured for our samples do not achieve the maximum methane adsorption uptake at 35 bar. We therefore use 50 bar as our reference maximum pressure to discuss and compare total methane uptakes.

The gravimetric and volumetric total adsorption, $G_{\text {tot }}$ and $V_{\text {tot }}$ respectively, were converted from experimentally measured excess methane adsorption using Eq. 2-4. The void volume and volumetric adsorption were both calculated using the samples' crystallographic density with the known implications we discussed earlier in the manuscript. Therefore, only MOF samples with known crystallographic densities are discussed here. The bulk density was measured for carbons based materials and polymers as they do not share the same crystalline platform as MOFs.

The gravimetric total amount of methane adsorbed increases with increasing surface area and 
micoropore volume as shown in Fig. 4 (a) and Fig. S3, similarly to the plots for the $G_{\text {max.ex }}$ in Fig. $1(a)$ and (b). Among all of the MOFs measured, $\mathrm{PCN}-6^{\prime}$ with the highest $G_{\text {max.ex }}$ of $0.182 \mathrm{~g} \cdot \mathrm{g}^{-1}$ also reaches the highest $G_{\text {tot }}$ of $0.272 \mathrm{~g} . \mathrm{g}^{-1}$ at 50 bar. Some data points change their relative positions significantly in Fig. 4 (a) and Fig. S3 comparing to those in Fig. 1(a) and (b), due to the addition of other parameters in the calculation. For example, UMCM-3 has the fifth highest $G_{\text {tot }}$ of $0.199 \mathrm{~g} \cdot \mathrm{g}^{-1}$, with only an average $G_{\max . e x}$ of $0.126 \mathrm{~g} \cdot \mathrm{g}^{-1}$. The reasons could be: (1) some MOFs with higher $G_{\text {max.ex }}$ have lower $G_{e x}$ at 50 bar; and (2) the $\rho_{\text {crys }}$ of some MOFs can be very low.

The free volume, which could be the total pore volume $V_{p}$ obtained experimentally using $\mathrm{Ar}$ as the adsorbate or the void volume calculated by Eq. 3, and the crystallographic density are related properties of an adsorbent. Adsorbents with lower crystallographic density have higher free volume. There are obvious linear trends in the plots of $G_{\text {tot }}$ at 50 bar versus $V_{p}$ and $\rho_{\text {crys }}$ shown in Fig. 4 (b) and (c), respectively. Materials with low $\rho_{\text {crys }}$ and high $v_{p}$, show high $G_{\text {tot }}$ at 50 bar. In the case of UMCM-3 discussed above, it has the second highest $V_{p}$ and the second lowest $\rho_{\text {crys }}$ among the MOFs measured. The combination of these two lead to high gravimetric total methane uptake. That indicates the important influence of crystallographic density (total pore volume) to the gravimetric total methane uptake.

Volumetric total adsorption capacity has to be determined when considering these materials as potential adsorbents for adsorbed methane technology. Pressure vessels defined by their water volume are used for compressed natural gas and ultimately the adsorbents performances will be compared to the current benchmark system. In Fig. S4 (a) and (b), the volumetric total methane uptakes $V_{\text {tot }}$ at 50 bar 
of studied MOFs are plotted against the BET specific surface area and micropore volume $V_{m . p}$, respectively. At that stage, there is no clear relationship between the textural characteristics of the adsorbents and their $V_{\text {tot }}$ unlike what was observed for $G_{\text {tot }}$. The trends in capacity differ significantly, comparing to the $G_{\text {tot }}$ at 50 bar plots. In Fig. 5(a) and (b), materials with low $V_{p}$ and high $\rho_{\text {crys }}$ have high $V_{\text {tot }}$ at 50 bar generally. NOTT-119 with the highest $V_{\text {tot }}$ at 50 bar of 173.8 g. L $^{-1}$ has the highest $\rho_{\text {crys }}, 1.327$ $\mathrm{mL} . \mathrm{g}^{-1}$. While PCN-6' with the highest $G_{\text {tot }}$ of $0.272 \mathrm{~g} \cdot \mathrm{g}^{-1}$ has relatively low $V_{\text {tot }}\left(79.4 \mathrm{~g} \cdot \mathrm{L}^{-1}\right.$, lower than the average value) because of its low $\rho_{\text {crys }}\left(0.292 \mathrm{~mL} \cdot \mathrm{g}^{-1}\right)$. These plots highlight that the crystallographic density (or bulk density) of an adsorbent, comparing to surface area and micropore volume, is the most critical factor to reach significant volumetric total methane uptake capacity. In fact, in real applications, bulk density, which is related to but cannot be directly reflected from crystallographic density, mainly affects the volumetric total capacity. The actual volumetric capacities based on packing densities of the MOFs may be very different from our current results, in term of both numbers and trends in those plots. One must keep in mind that using crystallographic densities lead to overestimated $V_{\text {tot }}$, as ideal case scenarios.

\subsection{Comparison of deliverable methane capacity (50-4.5 bar)}

The deliverable methane capacity is defined as the amount of methane that is desorbed from the adsorption service pressure to a specific desorption pressure. This is actually the most essential parameter in the evaluation of adsorbents for the adsorbed natural gas technology vehicular applications. The methane uptake at low pressure shall be considered in addition to the previous discussions. 5 bar is usually cited as a minimum desorption pressure in order to maintain a sufficient 
inlet pressure to fuel the engine. However, a lower minimum desorption pressure would allow for a higher deliverable capacity and longer vehicle driving range. $50-4.5$ bar was selected as the usable pressures range in this section.

The plots of $G_{e x}$ at 4.5 bar versus BET surface area are presented in Fig. S5(a). Interestingly, most of samples with relatively high methane uptake at 4.5 bar (higher than $0.04 \mathrm{~g} . \mathrm{g}^{-1}$ ) are copper centered MOFs, regardless their surface areas. This is consistent with the statement established previously in the literature; copper centers (normally $\mathrm{Cu}(\mathrm{II})$ ) are strong primary adsorption sites [12,22,31]. Under the experimental conditions, methane molecules occupy open copper sites first, and distribute unevenly in the adsorbent [31]. Comparing with MOFs having no strong adsorption sites, such as IRMOF-1 with $\mathrm{Zn}$ metal centers, the Cu centered MOFs generally have isotherms with steep initial slopes. Fig. 6(a) depicts the isotherms of IRMOF-1 (Zn), PCN-61 (Cu), PCN-11 (Cu) and PCN-307 (Cu). The maximum excess methane adsorptions of PCN-307 is comparable to that of IRMOF-1. Both PCN-61 and PCN-11 have lower $G_{\text {max.ex. }}$ On the other hand, all the Cu centered MOFs have higher $G_{e x}$ at low pressures (up to about 20 bar) than IRMOF-1 (Zn) does, suggesting that they have a higher adsorption energy.

Besides the effects from the $\mathrm{Cu}$ adsorption sites, other factors, such as the micropore shapes, pore size, and pore size distributions, could contribute to the adsorption energy. In term of pore size and pore size distributions, PCN-61 has similar average pore width (10.48 $\AA$ ) to IRMOF-1 (11.98 $⿱$ ), the isotherms shape differences are mainly due to the primary methane adsorption sites. However, both PCN-307 and PCN-11 have smaller average pore width, 7.93 and $7.55 \AA$, respectively. The micropore size distribution of PCN-307 is extremely narrow as described above. From the studies of carbon adsorbents, it has been 
established that highly microporous carbons have steep initial slopes to their uptakes, and therefore retain a larger proportion of the gas on delivery [32].

The methane adsorption at 4.5 bar causes the changes in the relative positions of the materials in volumetric deliverable capacities versus crystallographic densities plots (Fig. S5(b)), compared to what is observed in the $V_{\text {tot }}$ at 50 bar plots (Fig. 5 (b)). Taking the comparison of Co MOF-74 and Zr-mBTB as an example, despite the fact that Co MOF-74 has much higher $V_{\text {tot }}$ at 50 bar than Zr-mBTB does, Zr-mBTB has a higher deliverable methane uptake. Their isotherms are illustrated in Fig. 6(b). At 4.5 bar, the higher adsorption energy of Co MOF-75 results in much higher $V_{\text {tot }}$, comparing to that of Zr-mBTB. The volumetric deliverable capacity of Co MOF-75 is 38.8 g.L L $^{-1}$, only $37.1 \%$ of its 50 bar total capacity (104.4 g. $\left.\mathrm{L}^{-1}\right)$. In contrast Zr-mBTB has $65.6 \mathrm{~g} \cdot \mathrm{L}^{-1}$ deliverable capacity, $83 \%$ of its 50 bar total capacity $\left(79 \mathrm{~g} \cdot \mathrm{L}^{-1}\right)$.

The discussion in this section illustrates the importance of optimizing the methane adsorption energy when designing adsorbents, specifically MOFs. Optimal micropore size, narrow micropore size distribution, and strong methane adsorption sites can help increase the adsorption energy and ultimately the material's overall performance. However, to achieve a good deliverable capacity, those parameters all have to be optimized to avoid retaining too much methane at low desorption pressure.

\section{Conclusion}

With detailed comparisons of the methane adsorption capacity and physical properties of 52 adsorbents, we have shown that the BET SSA and micropore volume of an adsorbent basically determine its maximum gravimetric excess methane adsorption capacity at $298 \mathrm{~K}$ and up to 70 bar, irrespective of 
the chemistry and functionalities of materials. In addition, the capacity also depends on the pore size distributions. Narrow pore size distribution with optimum pore width improves the gravimetric maximum excess methane adsorption. The pressure at which the material reaches its maximum excess methane adsorption relates to the total pore volume, also irrespective of the various materials or metal centers. The impacts from different metal centers are relatively low on adsorption enthalpy and capacity at moderate pressures (20- 70 bar). Both the gravimetric and volumetric total methane capacities at 50 bar closely relate to the packing density of the adsorbents. For the volumetric capacity, which can be directly linked to the driving range of a vehicle, packing density is the major factor among all the physical properties. In this paper and most of published studies, crystallographic density is used instead of actual packing density of MOF sample for the volumetric total gas uptake determination. The total volumetric capacities calculated this way end up being much higher than those in reality. The capacity change due to different open metal centers as primary methane adsorption sites of different materials can be observed obviously at relatively low pressure $(<5$ bar $)$. However, the methane adsorption energy has to be thought of carefully, since it may decrease the deliverable capacity, which is actually most directly related to NG vehicular applications.

In this study, PCN -307, PCN-70, PCN-305, and NOTT-119 all display deliverable storage uptakes higher than $100 \mathrm{~g} \cdot \mathrm{L}^{-1}$, with NOTT-119 having the highest uptake of $140.7 \mathrm{~g} \cdot \mathrm{L}^{-1}$. Interestingly, these MOFs are all $\mathrm{Cu}$ based MOFs which are in general moisture sensitive, adding difficulties to practical use. The current achieved deliverable capacity would still require improvement as well. To design MOFs as better NG adsorbents, we conclude that large surface area and micropore volume with optimum pore size 
distributions are desired. Many studies have been focusing on those parameters, however, more work has to be done to understand the influence and to improve the packing density of adsorbents. 


\section{Acknowledgement}

This research was financed in part by the U.S. Department of Energy, Basic Energy Sciences, grant DE-AC02-06CH11357 through the Center for Electrical Energy Storage, an Energy Frontier Research Center. The Texas A\&M University, University of Nottingham, University of California Berkeley, and University of Michigan are acknowledged for their help in providing the materials. 


\section{Reference}

[1] K.R. Matranga, A.L. Myers, E.D. Glandt, Storage of natural gas by adsorption on activated carbon, Chem. Eng. Sci. 47 (1992) 1569-1579.

[2] D.F. Quinn, J.A. Macdonald, Natural gas storage, Carbon 30 (1992) 1097-1103.

[3] J. A. Mason, M. Veenstra, J.R. Long, Evaluating metal-organic frameworks for natural gas storage, Chem. Sci. 5 (2014) 32.

[4] V.C. Menon, S. Komarneni, Porous adsorbents for vehicular natural gas storage : a review, J. Porous Mater. 5 (1998) 43-58.

[5] D. Lozano-Castelló, J. Alcañiz-Monge, M.A. DE LA Casa-Lillo, D. Cazorla-Amorós, A. Linares-Solano, Advances in the study of methane storage in porous carbonaceous materials, Fuel. 81 (2002) 1777-1803.

[6] R.F. Cracknell, P. Gordon, K.E. Gubbins, Influence of pore geometry on the design of microporous materials for methane storage, J. Phys. Chem. 97 (1993) 494-499.

[7] R.J. Remick, A.J. Tiller, Advanced methods for low-pressure storage of CNG, in: Proc. Symp. Nonpetroleum Veh. Fuels V, 1985: pp. 105-119.

[8] T.D. Burchell, Carbon materials for advanced technologies, New York, 1999.

[9] D. Elliott, T. Topaloglu, The development of new adsorbent materials for the storage of natural gas on-board vehicles, in: Conf. Proc. Gaseous Fuels Transp. I, 1986: pp. 489-504.

[10] R.E. Morris, P.S. Wheatley, Gas storage in nanoporous materials., Angew. Chemie Int. Ed. 47 (2008) 4966-4981.

[11] S. Ma, H.-C. Zhou, Gas storage in porous metal-organic frameworks for clean energy applications, Chem. Commun. 46 (2010) 44-53.

[12] Y. He, W. Zhou, T. Yildirim, B. Chen, A series of metal-organic frameworks with high methane uptake and an empirical equation for predicting methane storage capacity, Energy Environ. Sci. 6 (2013) 2735.

[13] Y. Peng, G. Srinivas, C.E. Wilmer, I. Eryazici, R.Q. Snurr, J.T. Hupp, et al., Simultaneously high gravimetric and volumetric methane uptake characteristics of the metal-organic framework NU-111, Chem. Commun. 49 (2013) 2992-2994. 
[14] T. A. Makal, J.-R. Li, W. Lu, H.-C. Zhou, Methane storage in advanced porous materials., Chem. Soc. Rev. 41 (2012) 7761-79.

[15] J.W. Patrick, Porosity in carbons: characterization and applications, Edward Arnold, New York, 1995.

[16] J. Alcañiz-Monge, M.A. DE LA Casa-Lillo, D. Cazorla-Amorós, A. Linares-Solano, Methane storage in activated carbon fibres, Carbon 35 (1997) 291-297.

[17] C.D. Wood, B. Tan, A. Trewin, F. Su, M.J. Rosseinsky, D. Bradshaw, et al., Microporous organic polymers for methane storage, Adv. Mater. 20 (2008) 1916-1921.

[18] D. Yuan, W. Lu, D. Zhao, H.-C. Zhou, Highly stable porous polymer networks with exceptionally high gas-uptake capacities., Adv. Mater. 23 (2011) 3723-3725.

[19] D. Lozano-Castelló, D. Cazorla-Amorós, a Linares-Solano, D.. Quinn, Influence of pore size distribution on methane storage at relatively low pressure: preparation of activated carbon with optimum pore size, Carbon 40 (2002) 989-1002.

[20] P.D.C. Dietzel, V. Besikiotis, R. Blom, Application of metal-organic frameworks with coordinatively unsaturated metal sites in storage and separation of methane and carbon dioxide, J. Mater. Chem. 19 (2009) 7362-7370.

[21] W. Zhou, H. Wu, M.R. Hartman, T. Yildirim, Hydrogen and methane adsorption in metal-organic frameworks: a high-pressure volumetric study, J. Phys. Chem. C. 111 (2007) 16131-16137.

[22] H. Wu, W. Zhou, T. Yildirim, High-capacity methane storage in metal - organic frameworks M2(dhtp): the important role of open metal sites, J. Am. Chem. Soc. 131 (2009) 4995-5000.

[23] C.E. Wilmer, M. Leaf, C.Y. Lee, O.K. Farha, B.G. Hauser, J.T. Hupp, et al., Large-scale screening of hypothetical metal-organic frameworks, Nat Chem. 4 (2012) 83-89.

[24] J.L. Mendoza-Cortés, S.S. Han, H. Furukawa, O.M. Yaghi, W. a Goddard, Adsorption mechanism and uptake of methane in covalent organic frameworks: theory and experiment., J. Phys. Chem. A. 114 (2010) 10824-33.

[25] D.A. Gómez-Gualdrón, C.E. Wilmer, O.K. Farha, J.T. Hupp, R.Q. Snurr, Exploring the limits of methane storage and delivery in nanoporous materials, J. Phys. Chem. C. 118 (2014) 6941-6951.

[26] H. Frost, T. Düren, R.Q. Snurr, Effects of Surface Area, Free Volume, and Heat of Adsorption on Hydrogen Uptake in Metal - Organic Frameworks, J. Phys. Chem. B. 110 (2006) 9565-9570. 
[27] T. Düren, L. Sarkisov, O.M. Yaghi, R.Q. Snurr, Design of new materials for methane storage, Langmuir. 20 (2004) 2683-2689.

[28] S. Wang, Comparative Molecular Simulation Study of Methane Adsorption in Metal - Organic Frameworks, Energy \& Fuels. 21 (2007) 953-956.

[29] F. Rouquerol, J. Rouquerol, K.S.W. Sing, P. Llewellyn, G. Maurin, Adsorption by powders and porous solids: principles, methodology and applications, 2nd ed., Academic press, 2014.

[30] E. Poirier, A. Dailly, On the nature of the adsorbed hydrogen phase in microporous metal-organic frameworks at supercritical temperatures, Langmuir. 25 (2009) 12169-12176.

[31] H. Wu, J.M. Simmons, Y. Liu, C.M. Brown, X.-S. Wang, S. Ma, et al., Metal-organic frameworks with exceptionally high methane uptake: where and how is methane stored?, Chem. - A Eur. J. 16 (2010) 5205-5214.

[32] R.W. Judd, D.T. Gladding, R.C. Hodrien, D.R. Bates, J.P. Ingram, M. Allen, The use of adsorbed natural gas technology for large scale storage, in: Prepr. Symp. Am Chem S O C. Div Fuel Chem, ACS, Vol. 43, no.3, 1998: pp. 575-579. 


\section{Figures}
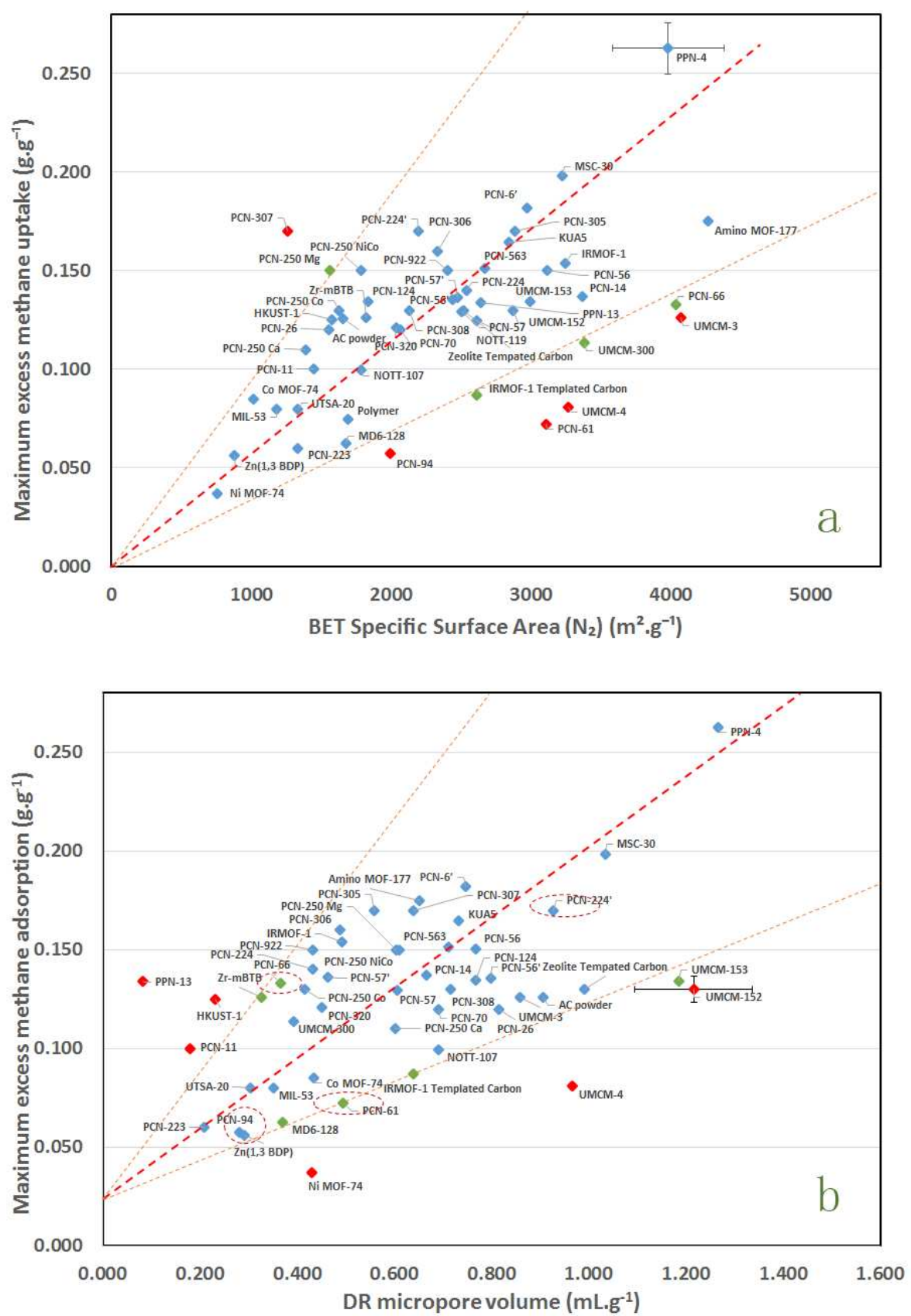

Fig. 1 Plots of maximum excess methane uptake versus BET Specific surface area (a), micropore volume

(b) for all materials. Error bars equivalent to $10 \%$ for $\mathrm{X}$ and $5 \%$ for $\mathrm{y}$, as represented on PPN-4 in (a), and

UMCM-152 in (b), respectively. 


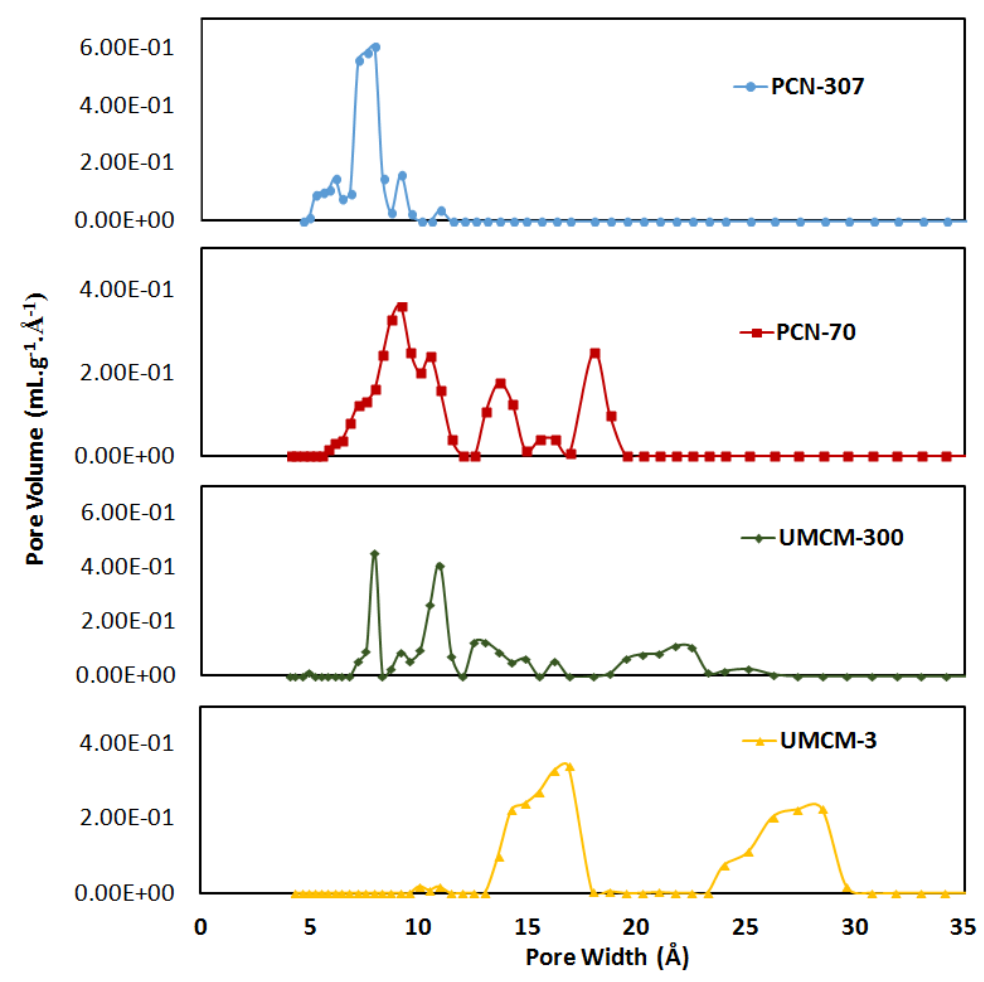

Fig. 2 Measured pore size distributions for PCN-307, PCN-70, UMCM-300, and UMCM-3.

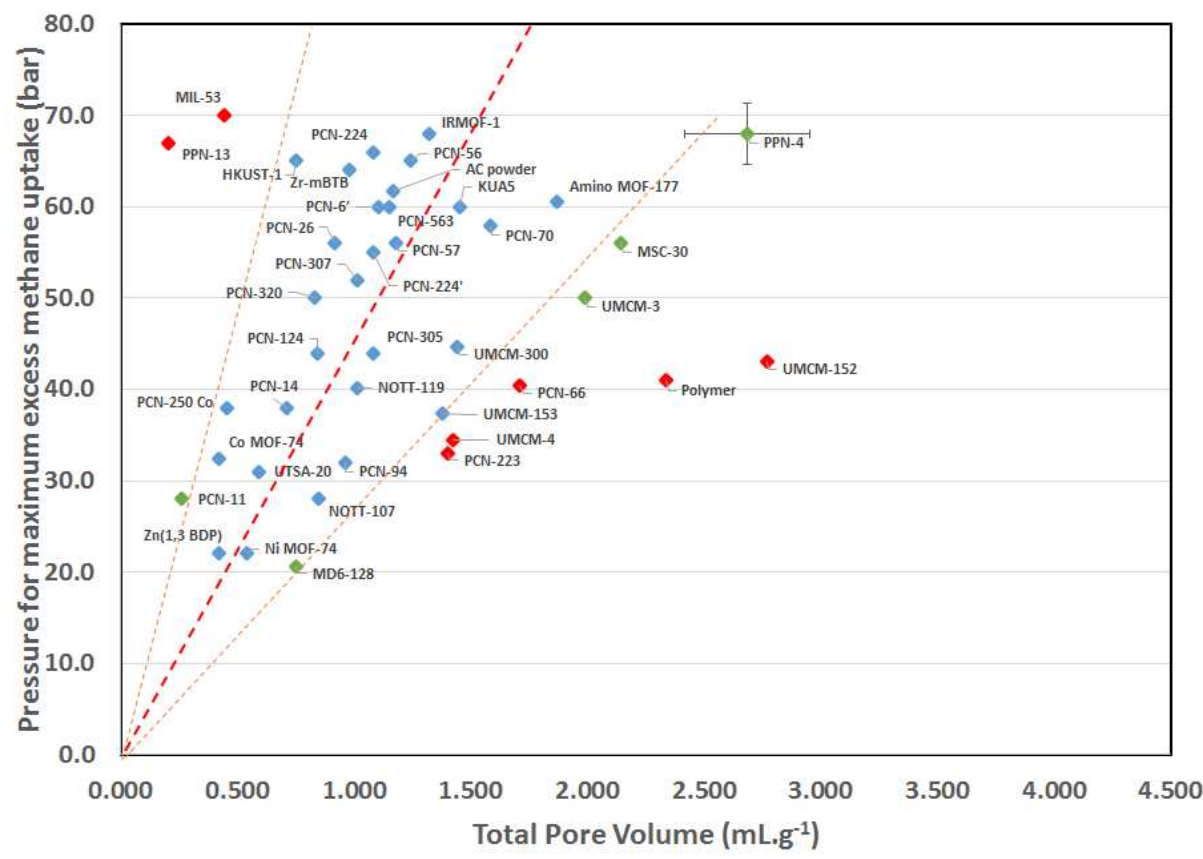

Fig. 3 Plots of $P_{\max \text { ex }}$ versus total pore volume for all materials. Error bars equivalent to $10 \%$ for $X$ and $5 \%$ for $\mathrm{y}$, as represented on PPN-4. 

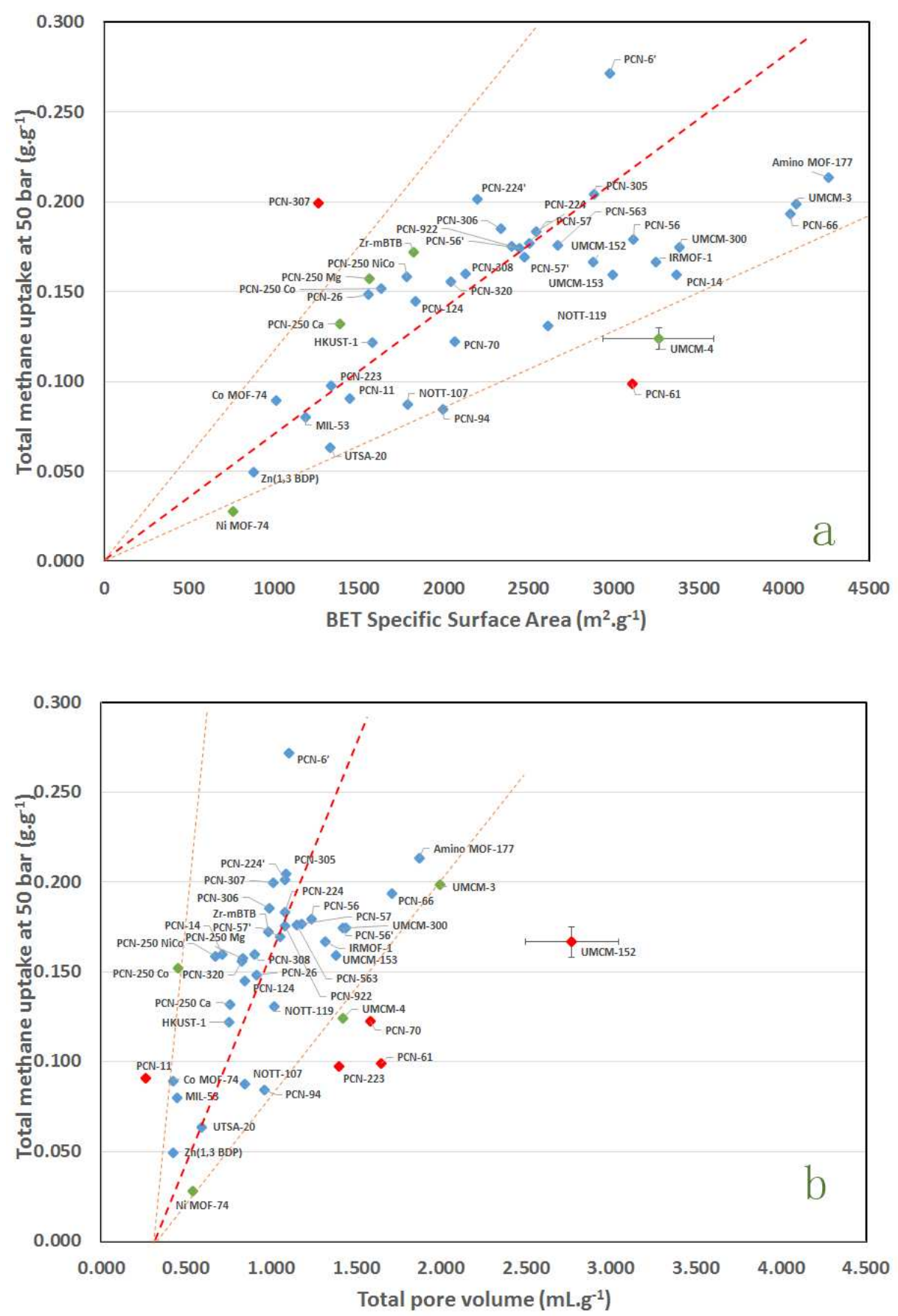


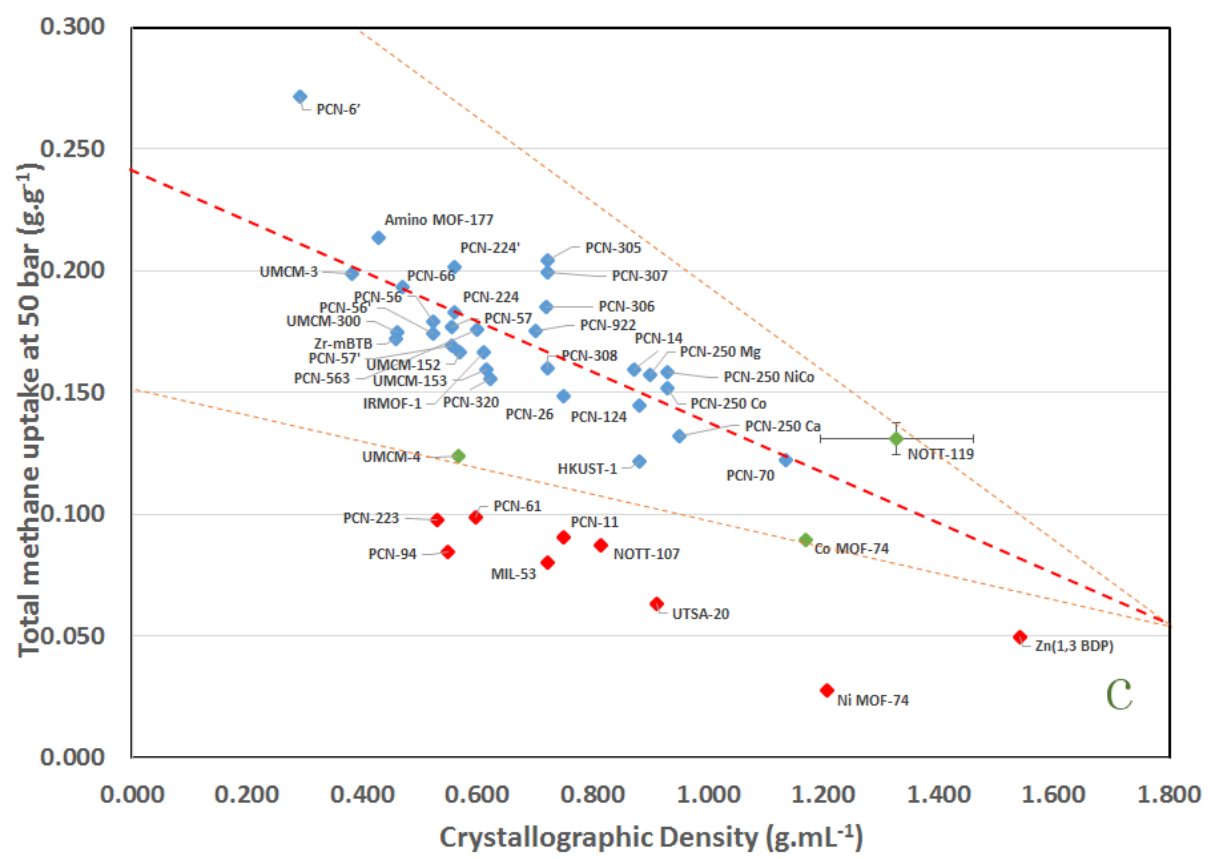

Fig. 4 Plots of gravimetric total methane uptake at 50 bar versus BET specific surface area (a), total pore volume (b), and crystallographic density (c) for all materials. Error bars equivalent to $10 \%$ for $\mathrm{X}$ and $5 \%$ for $y$, as represented on UMCM-4 in (a), UMCM-152 in (b), and NOTT-119 in (c), respectively.

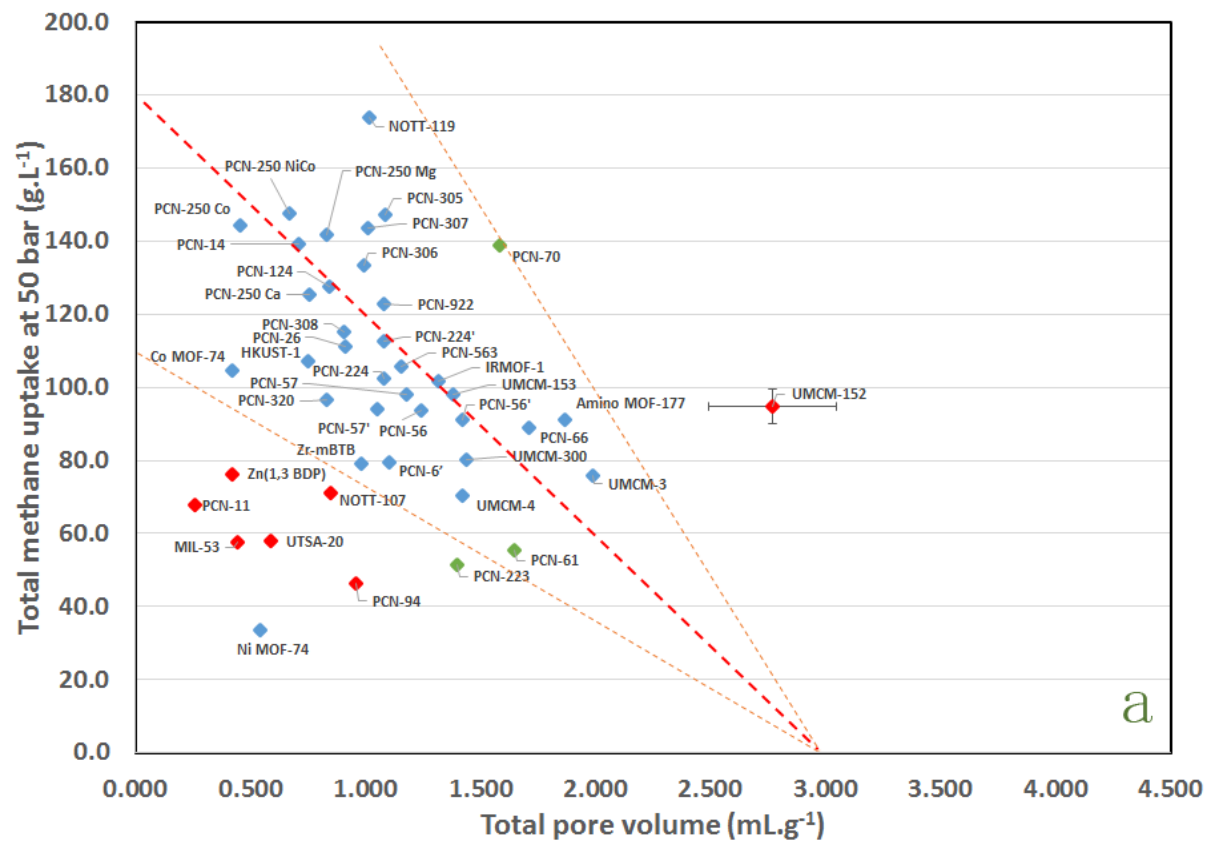




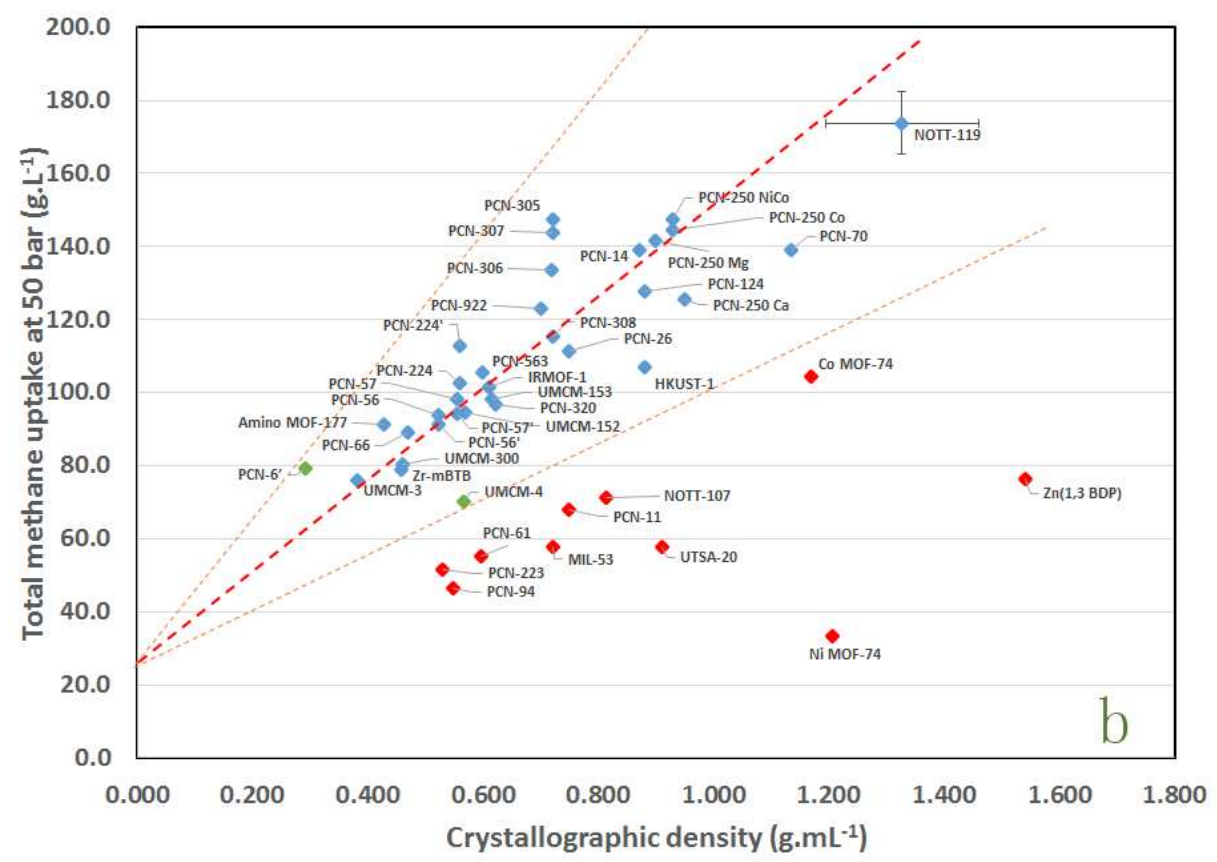

Fig. 5 Plots of volumetric total methane uptake at 50 bar versus total pore volume (a), and crystallographic density (b) for all materials. Error bars equivalent to $10 \%$ for $\mathrm{X}$ and $5 \%$ for $\mathrm{y}$, as represented on UMCM-152 in (a), and NOTT-119 in (b), respectively. 

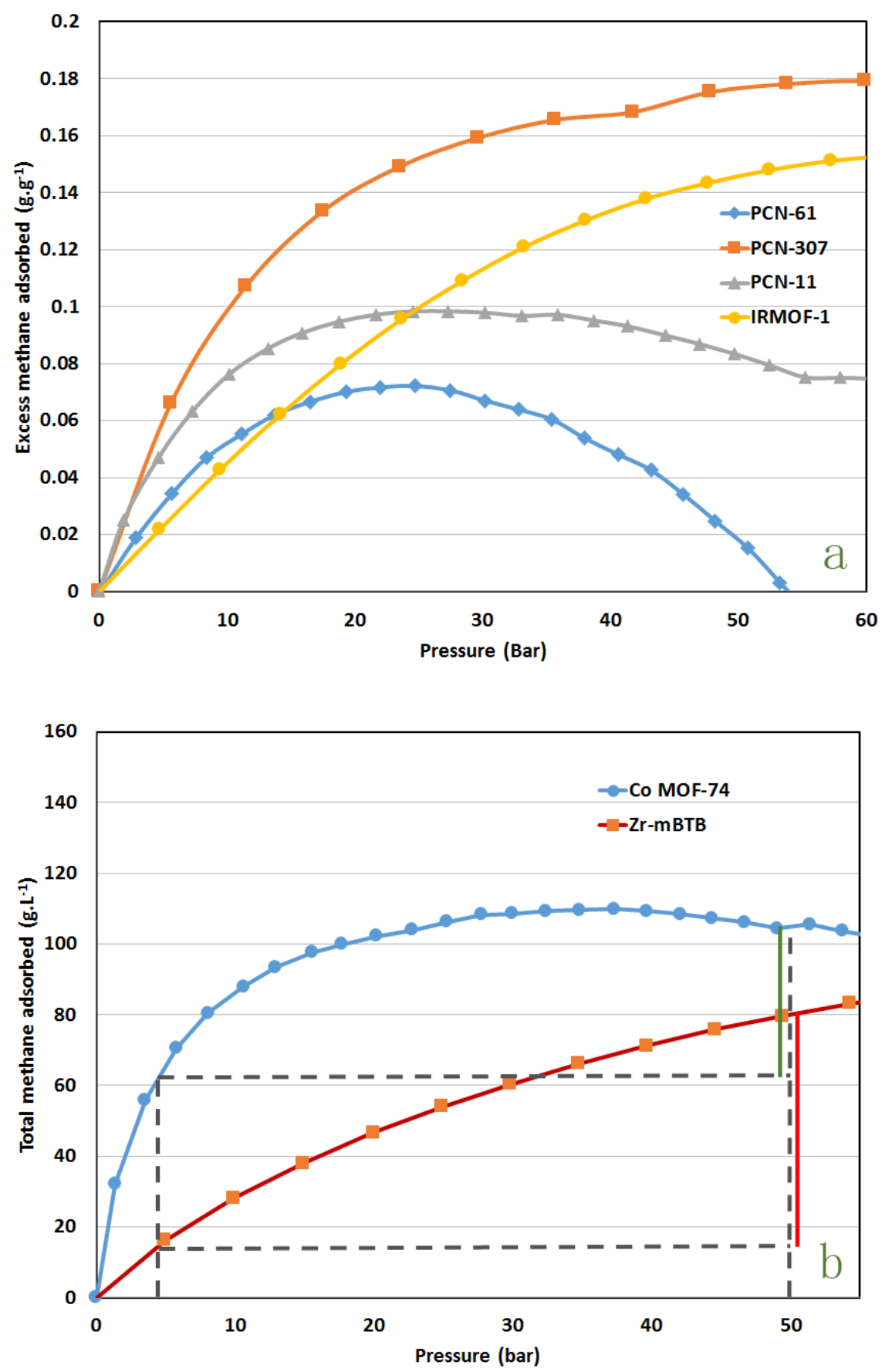

Fig. 6 (a) excess methane adsorption at 298K on PCN-61, PCN-307, PCN-11, and IRMOF-1, and (b) volumetric total methane adsorption at $298 \mathrm{~K}$ on Co MOF-74 and Zr-mBTB. 